\title{
Multi-frequency GMRT observations of the Hı regions S 201, S 206, and S 209
}

\section{Galactic temperature gradient}

\author{
A. Omar ${ }^{1}$, J. N. Chengalur ${ }^{2, \star}$, and D. Anish Roshi ${ }^{3, \star \star}$ \\ 1 Raman Research Institute, C.V. Raman Avenue, Bangalore 560080, India \\ 2 National Centre for Radio Astrophysics, Post Bag 3, Ganeskhind, Pune 411007, India \\ 3 National Radio Astronomical Observatory, Green Bank, West Virginia, 24944-0002, USA
}

Received 12 November 2001 / Accepted 28 August 2002

\begin{abstract}
We present radio continuum images of three Galactic Hir regions, S 201, S 206, and S 209 near 232, 327, and $610 \mathrm{MHz}$ using the Giant Meterwave Radio Telescope (GMRT). The GMRT has a mix of short and long baselines, therefore, even though the data have high spatial resolution, the maps are still sensitive to diffuse extended emission. We find that all three $\mathrm{H}_{\text {II }}$ regions have bright cores surrounded by diffuse envelopes. We use the high resolution afforded by the data to estimate the electron temperatures and emission measures of the compact cores of these $\mathrm{H}_{\text {II }}$ regions. Our estimates of electron temperatures are consistent with a linear increase of electron temperature with Galacto-centric distance for distances up to $\sim 18 \mathrm{kpc}$ (the distance to the most distant $\mathrm{H}$ II region in our sample).
\end{abstract}

Key words. ISM: H II regions - ISM: individual objects: S 201, S 206, S 209 - radio continuum: ISM

\section{Introduction}

A number of studies have indicated that the electron temperature $T_{\mathrm{e}}$ of $\mathrm{H}$ II regions increases with increasing Galacto-centric distance (e.g. Deharveng et al. 2000 and references therein). This effect is attributed to a decrease in heavy elements abundances with Galacto-centric distance. A low metal abundance leads to less effective cooling and consequently higher electron temperature. These studies are based either on estimates of $T_{\mathrm{e}}$ from radio recombination lines (RRLs) (which in turn depend on corrections for departures from local thermodynamic equilibrium (LTE) and for collisional broadening effects), or estimates based on line strengths of the forbidden line transitions of oxygen [O III] $\lambda \lambda 4363,5007$ (which are strongly dependent on temperature variations, if any, over the observed volume). Further, most of these studies are based on observations of $\mathrm{H}_{\text {II }}$ regions with Galacto-centric distances $R_{\mathrm{G}} \leq 15 \mathrm{kpc}$ with very few measurements of $T_{\mathrm{e}}$ beyond $15 \mathrm{kpc}$. Consequently most determinations of metalicities of the outer galaxy $\mathrm{H}$ in regions are based on values of $T_{\mathrm{e}}$ taken from an extrapolation of the observed gradient in temperature up to about $15 \mathrm{kpc}$ (e.g., Deharveng et al. 2000). Since the O/H ratio (a commonly used indicator of metal abundance) depends sensitively on $T_{\mathrm{e}}$,

Send offprint requests to: A. Omar, e-mail: aomar@rri.res.in

* e-mail: chengalu@ncra.tifr.res.in

$\star \star$ e-mail: aroshi@gb.nrao.edu metalicities of the outer galaxy $\mathrm{H}$ II regions are poorly constrained. In view of this, it is important to get independent estimates of the electron temperatures of $\mathrm{H}$ II regions in the outer galaxy.

An independent measurement of $T_{\mathrm{e}}$ can be obtained from radio continuum observations. The ionized material in $\mathrm{H}$ II regions emits radio continuum through free-free emission. At sufficiently low radio frequencies where the nebula is optically thick $(\tau>>1)$, the emergent radiation is a black body spectrum, and therefore, the observed brightness temperature is equal to the electron temperature $T_{\mathrm{e}}$. On the other hand, at sufficiently high radio frequencies, where the optical depth $\tau$ of thermal electrons is low $(\tau<<1)$, the observed brightness is proportional to the emission measure of the nebula. Most of the available radio maps for $\mathrm{H}$ II regions are at high radio frequencies (i.e. above 1.4 GHz, e.g., Fich 1993; Balser et al. 1995). These maps show that $\mathrm{H}$ II regions often have a bright core with several knots surrounded by an extended envelop of diffuse emission. These core-envelope structures of $\mathrm{H}_{\text {II }}$ regions imply that accurate measurement of $T_{\mathrm{e}}$ from low radio frequency observations requires high angular resolution, since, often only bright compact cores will be optically thick at frequencies of a few hundred MHz. This study presents an analysis of the lowfrequency GMRT observations of three Galactic diffuse H II regions spanning Galacto-centric distances up to $18 \mathrm{kpc}$.

The GMRT is an ideal telescope for these observations since it operates at several low radio frequency bands, viz., 
$150,232,327,610$, and $1420 \mathrm{MHz}$ and also it has a hybrid configuration which makes it sensitive to both diffuse emission (on scales up to $\sim 45^{\prime}$ at $232,30^{\prime}$ at 327 , and $17^{\prime}$ at $610 \mathrm{MHz}$ ) while also having the resolution $\left(\sim 15^{\prime \prime}\right.$ at $232,10^{\prime \prime}$ at 327 , and $6^{\prime \prime}$ at $610 \mathrm{MHz}$ ) to resolve the compact cores.

\section{Observations}

The observations were carried out during the period of August to December, 1999 at three frequency bands, viz., 232, 327, and $610 \mathrm{MHz}$. The GMRT has a "Y" shaped hybrid configuration of antennas with six antennas along each of the three arms and twelve antennas randomly placed in a compact arrangement near the centre of "Y" (for details, see Swarup et al. 1991). The compact array at the centre is about a kilometer across and is generally referred as the "central square". Baselines in the central square (shortest baseline $\sim 100 \mathrm{~m}$ ) provide sensitivity to diffuse large scale emission, while baselines involving arm antennas (longest baseline $\sim 25 \mathrm{~km}$ ) provide high angular resolution. The GMRT was in its commissioning phase during our observations, and due to various debugging and maintenance activities not all 30 antennas were available for observations. The observations were carried out with typically 20 to 25 antennas in different observing sessions.

The data were recorded in the default correlator mode which produces visibilities in 128 channels over a user selectable bandwidth in multiples of 2 starting from $62.5 \mathrm{kHz}$ and up to $16 \mathrm{MHz}$. The observational parameters are summarized in Table 1. The observations near 610 and $327 \mathrm{MHz}$ were made using the full $16 \mathrm{MHz}$ bandwidth while observations near $232 \mathrm{MHz}$ were made with a bandwidth of $2 \mathrm{MHz}$ centered at a frequency around which least local interference has been detected in the past observations. The images at all frequencies are however made using data from only one channel which corresponds to a bandwidth of $125 \mathrm{kHz}$ at 327 and $610 \mathrm{MHz}$, and $15.6 \mathrm{kHz}$ at $232 \mathrm{MHz}$. This restriction was partly because of a crunch in disk storage at the time when these data were taken, and partly because dynamic range limitations at the GMRT at the time we took the data meant that the increase in bandwidth did not result in a proportionate increase in sensitivity. At each frequency band, we observed the source for about 8-10 hours, primarily in order to have a good $(u, v)$ coverage.

For all the observations, the source $3 \mathrm{C} 48$ was used as the primary flux calibrator. The flux density of $3 \mathrm{C} 48$ at each frequency was estimated using the Baars et al. (1977) flux densities of standard VLA calibrators. The phase and amplitude gains of antennas were derived from observations of a secondary calibrator at intervals of $45 \mathrm{~min}$. For observations on S 206 and S 209, 3C 119 was used as a secondary calibrator while $0107+562$ was used as a secondary calibrator for observations on S 201. Both 3C 119 and 0107+562 are standard VLA calibrators. The fluxes of secondary calibrators were determined via boot-strapping the fluxes of the primary calibrator $3 \mathrm{C} 48$.

The data were carefully checked for interference or other problems. At 232 and $327 \mathrm{MHz}$, a few short baselines were found to be corrupted, possibly by interference, and were removed. The data at $610 \mathrm{MHz}$ were found to be free from any interference. Data reduction was done in classic AIPS. The calibrated data were Fourier transformed using appropriate $(u, v)$ ranges, tapers and weights to make different images, some of which are sensitive to large scale structures, and others which have the maximum possible angular resolution. These images were deconvolved using the "CLEAN" algorithm as implemented in AIPS task "IMAGR". The final gains of the antennas were fixed using several iterations of self-calibration.

The variations in system temperatures of GMRT antennas are currently not routinely monitored during observations. The system temperature at $610 \mathrm{MHz}$ was measured both toward the absolute flux calibrator 3C 48 and the target source by firing the noise calibration diodes. For 327 and $232 \mathrm{MHz}$ images, the system temperature toward 3C 48 and target source were obtained using interpolated values of sky temperature from $408 \mathrm{MHz}$ all-sky map of Haslam et al. (1982). A correction factor equal to the ratio of the system temperature toward the target source and 3C 48 was applied in the deconvolved image. The deconvolved images were finally corrected for the primary beam attenuation, assuming a Gaussian shape for the primary beam. The half power points (HPBW) of the primary beam of GMRT antenna are estimated as $1.85,1.35$, and 0.72 degree for 232, 327 and $610 \mathrm{MHz}$ respectively.

\section{Results}

Images of S 201 are shown in Figs. 1a-c. S 201, $(l=$ $138.48, b=1.64$; also known as IC 1848), at a Galacto-centric distance of $10.5 \pm 1 \mathrm{kpc}$, is believed to be excited due to a single star of spectral type O9.5 (Mampaso et al. 1989). High resolution $\left(\sim 5^{\prime \prime}\right) 15 \mathrm{GHz}$ radio continuum image reveals a bright arc like core with multiple peaks of emission (Felli et al. 1987). The $616 \mathrm{MHz}$ GMRT low resolution image (Fig. 1c) traces diffuse emission extending up to $\sim 5^{\prime}$ which is consistent with the $1.4 \mathrm{GHz}$ VLA image of Fich (1993). The high resolution $231 \mathrm{MHz}$ GMRT image (Fig. 1b) shows the core to be a complex structure consisting of several unresolved compact sources. The diffuse nebulosity extending toward the west of the core in the $231 \mathrm{MHz}$ image (Fig. 1b) is consistent with the $15 \mathrm{GHz}$ radio image of Felli et al. (1987).

Images of S 206 are shown in Figs. 1d-f. S 206, $(l=$ $150.74, b=-0.75$; also known as NGC 1491), is an evolved $\mathrm{H}$ II region at a Galacto-centric distance of $11.1 \mathrm{kpc}$ (Deharveng et al. 2000). The excitation is believed to be provided by a single O5 star (Crampton \& Fisher 1974). The $5 \mathrm{GHz}$ radio continuum image shows a classic blister type morphology (Fig. 4 in Deharveng et al. 1976) as described in Icke et al. (1980). Our high resolution images at $613 \mathrm{MHz}$ (Fig. 1d), $236 \mathrm{MHz}$ (Fig. 1e) as well as $328 \mathrm{MHz}$ (not shown) show good correspondence to the $5 \mathrm{GHz}$ image of Deharveng et al. (1976). The low resolution $328 \mathrm{MHz}$ image (Fig. 1f) shows a large low intensity envelope surrounding the core emission.

Images of S 209 are shown in Figs. 1g-i. S 209 ( $l=151.6$, $b=-0.24$; also known as RAFGL 550) is one of the most distant $\left(R_{\mathrm{G}}=17.7 \mathrm{kpc}\right.$, Deharveng et al. 2000) Galactic $\mathrm{H}$ II region. Although the mean size of $\mathrm{H}_{\text {II }}$ regions decreases with increasing Galacto-centric distance (Fich \& Blitz 1984), S 209 is unusual in that it has a very large size $(\sim 50 \mathrm{pc})$ 
Table 1. Observational details.

\begin{tabular}{lccccc}
\hline \hline $\begin{array}{l}\text { Field Centre } \\
\text { RA } \\
(\mathrm{B} 1950)\end{array}$ & $\begin{array}{c}\text { Field Centre } \\
\text { Dec } \\
(\mathrm{B} 1950)\end{array}$ & Frequency & $\begin{array}{c}\text { Duration of } \\
\text { observation } \\
(\text { Hours })\end{array}$ & $\begin{array}{c}\text { Range of } \\
\text { baselines } \\
(\mathrm{k} \lambda)\end{array}$ & $\begin{array}{c}\text { rms noise in } \\
\text { the image } \\
\left(\mathrm{mJy} \mathrm{beam}^{-1}\right)\end{array}$ \\
\hline $02^{\mathrm{h}} 59^{\mathrm{m}} 12^{\mathrm{s}}$ & $60^{\circ} 17^{\prime} 00^{\prime \prime}$ & 231 & 8 & $0.05-15$ & 2.5 \\
& & 616 & 8 & $0.09-25$ & 1.2 \\
$03^{\mathrm{h}} 59^{\mathrm{m}} 24^{\mathrm{s}}$ & $51^{\circ} 11^{\prime} 00^{\prime \prime}$ & 236 & 9 & $0.05-18$ & 7.4 \\
& & 328 & 10 & $0.06-27$ & 3.0 \\
& & 613 & 10 & $0.09-49$ & 1.2 \\
$04^{\mathrm{h}} 07^{\mathrm{m}} 18^{\mathrm{s}}$ & $51^{\circ} 02^{\prime} 00^{\prime \prime}$ & 328 & 9 & $0.10-26$ & 2.0 \\
& & 613 & 10 & $0.10-50$ & 1.0 \\
\hline
\end{tabular}

Table 2. H II regions - results.

\begin{tabular}{ccccccc}
\hline \hline Name & $\begin{array}{c}\alpha, \delta(1950) \\
\mathrm{h} \mathrm{m}{ }^{\circ}, "\end{array}$ & $\begin{array}{c}\text { Frequency } \\
(\mathrm{MHz})\end{array}$ & $\begin{array}{c}\text { Flux } \\
(\mathrm{Jy})\end{array}$ & $\begin{array}{c}\text { Area } \\
\left(\operatorname{arcmin}^{2}\right)\end{array}$ & $\begin{array}{c}T_{\mathrm{e}} \\
(\mathrm{K})\end{array}$ & $\begin{array}{c}E M \\
\left(\mathrm{~cm}^{-6} \mathrm{pc}\right)\end{array}$ \\
\hline S 201 & 025920.1601610 & 231 & 0.78 & 16 & $7070 \pm 1100$ & $1.02( \pm 0.05) \times 10^{5}$ \\
& & 616 & 1.15 & 38 & & \\
S 206 & 035924.0511100 & 236 & 16.3 & 329 & $8350 \pm 1600$ & $3.93( \pm 0.40) \times 10^{5}$ \\
& & 328 & 18.2 & 347 & & \\
& & 613 & 20.0 & 350 & & \\
S 209 & & 236 & 13.4 & 267 & $10855 \pm 3670$ & $2.58( \pm 0.29) \times 10^{5}$ \\
& & 328 & 16.6 & 372 & & \\
\end{tabular}

for its Galacto-centric distance. The excitation is provided by a cluster of OB stars (Chini \& Wink 1984). Our high resolution $613 \mathrm{MHz}$ map (Fig. 1g) shows the core region to consist of an asymmetric, incomplete ring like structure. The high resolution image at $328 \mathrm{MHz}$ (not shown here) is morphologically very similar to the $613 \mathrm{MHz}$ image. The $236 \mathrm{MHz}$ image (Fig. 1h) while showing overall similarity to the $613 \mathrm{MHz}$ and $328 \mathrm{MHz}$ maps, does show some difference in the core region. We are unsure why this should be so. The low resolution $613 \mathrm{MHz}$ image (Fig. 1i) shows that this region too has an extremely large, low surface brightness envelope, which has also been seen at $2.7 \mathrm{GHz}$ image of Walmsley et al. (1975).

\section{Discussion}

We use these low frequency images to estimate electron temperatures and emission measures of the compact cores of the $\mathrm{H}$ II regions. If we approximate these cores to be homogeneous and spherically symmetric, then the flux $S$ is given by

$S=3.07 \times 10^{-2} T_{\mathrm{e}} v^{2} \Omega\left(1-\mathrm{e}^{-\tau(v)}\right)$

$\tau(v)=1.643 a \times 10^{5} v^{-2.1} E M T_{\mathrm{e}}^{-1.35}$

(Mezger \& Henderson 1967) where $S$ is the integrated flux density in $\mathrm{Jy}, T_{\mathrm{e}}$ is the electron temperature in Kelvin, $v$ is the frequency of observation in $\mathrm{MHz}, \tau$ is the optical depth, $\Omega$ is the solid angle subtended by the source in steradian, (which in this case, since the cores are unresolved, is taken to be the synthesized beam size), and $E M$ is the emission measure in $\mathrm{cm}^{-6} \mathrm{pc}$. The emission measure $E M$ is defined as $\int n_{\mathrm{e}}^{2} \mathrm{~d} l$; the integral being taken along the line of sight and averaged over the beam. $a$ is a correction factor which depends both upon the temperature and frequency. We have used an average value of $a$ as 0.98 (using Table 6 of Mezger \& Henderson 1967) for the frequency range $200-600 \mathrm{MHz}$ and $T_{\mathrm{e}} \sim 10000 \mathrm{~K}$. The $\mathrm{H}$ il region cores can be modeled by solving Eqs. (1) and (2) iteratively for different $E M$ and $T_{\mathrm{e}}$. The fitting procedure converges rapidly when observations at least two frequencies are available and the frequencies are such that the $\mathrm{H}$ II region is optically thick at one frequency and optically thin at the other.

We measured the peak flux densities of cores after convolving the images of a $\mathrm{H}$ il region at different frequencies to a common angular resolution (i.e. the source size $\Omega$ was taken to be $1.133 \times \theta_{\mathrm{a}} \times \theta_{\mathrm{b}}$, where $\theta_{\mathrm{a}}$ and $\theta_{\mathrm{b}}$ are the half power points of the common convolved beam). The best fit values for $T_{\mathrm{e}}$ and $E M$ as obtained from the fitting procedure described above are listed in Table 2, and the observed and model fluxes are plotted in Fig. 2. The columns in Table 2 are as follows. Column 1: Name of the H II region, Col. 2: Coordinates (right ascension, declination) of the core for which the electron temperature has been measured, Col. 3: The frequency of observation, Col. 4: Integrated flux of the entire $\mathrm{H}$ II region, Col. 5: Area over which radio emission is detected, and over which the flux has been integrated to get the value listed in Col. 4, Col. 6: Estimated electron temperature of the core, Col. 7: Estimated emission measure of the core.

The electron temperature of S 201 is estimated to be $7070 \pm$ $1100 \mathrm{~K}$ toward the peak radio emission. The earlier estimate for $T_{\mathrm{e}}$ toward $\mathrm{S} 201$ was $\sim 5000 \mathrm{~K}$ based on non-detection of [O III] $\lambda \lambda 4959,5007$ (Mampaso et al. 1989). The electron 

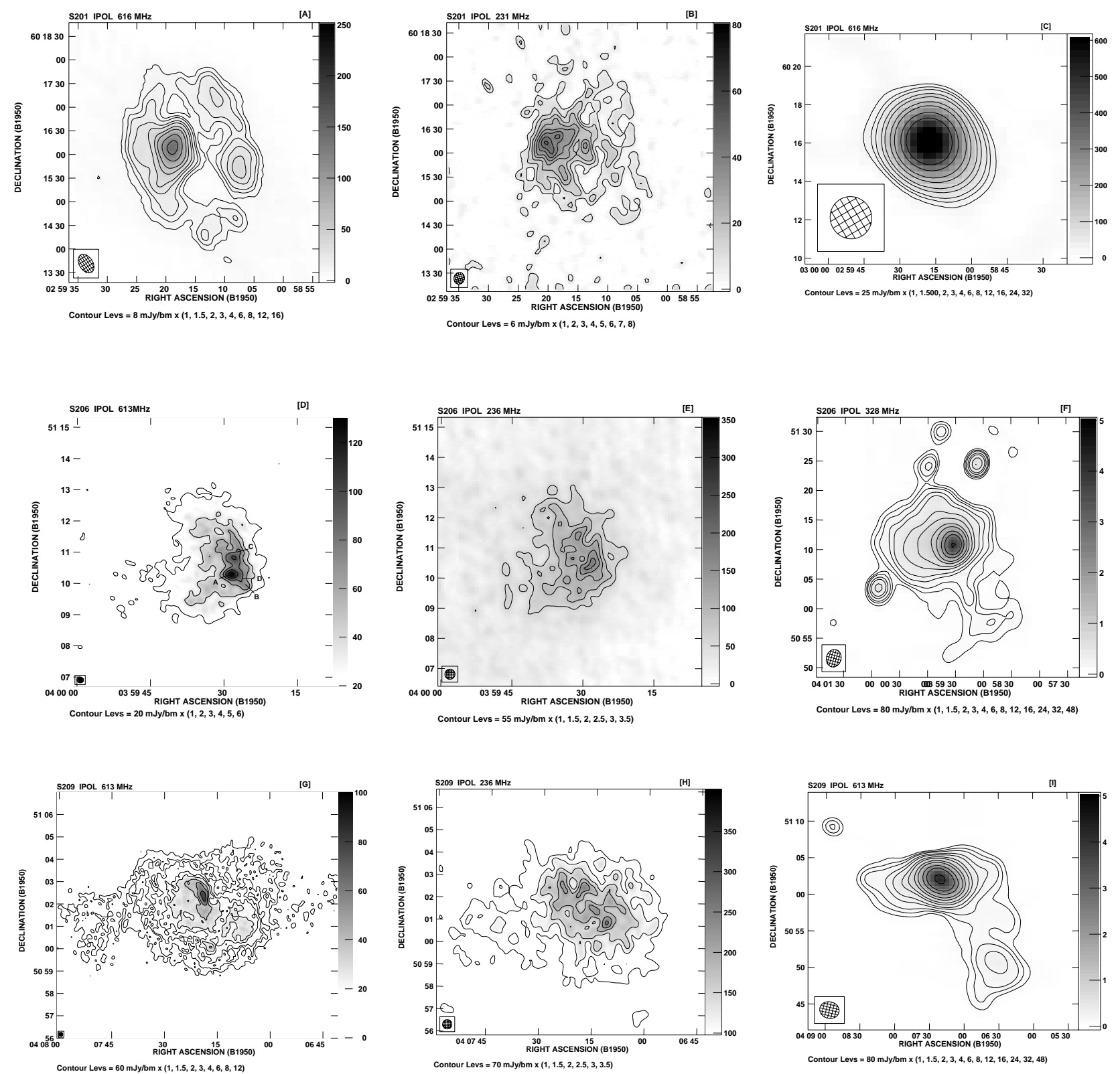

Fig. 1. a) S 201 at $616 \mathrm{MHz}$. The angular resolution is $26^{\prime \prime} \times 17^{\prime \prime}$. b) S 201 at $231 \mathrm{MHz}$. The angular resolution is $15^{\prime \prime} \times 13^{\prime \prime}$. c) S 201 at $616 \mathrm{MHz}$ made using $u-v$ range $0-1 \mathrm{k} \lambda$ only. The angular resolution is $133^{\prime \prime} \times 129^{\prime \prime}$. d) S 206 at $613 \mathrm{MHz}$. The angular resolution is $13^{\prime \prime} \times 11^{\prime \prime}$. The regions marked as A, B, C, \& D are from the $5 \mathrm{GHz}$ image of Deharveng et al. (1976). e) S 206 at $236 \mathrm{MHz}$. The angular resolution is $20^{\prime \prime} \times 20^{\prime \prime}$. f) $328 \mathrm{MHz}$ image of S 206 made using $u-v$ range $0-1 \mathrm{k} \lambda$ only. The synthesized beam is $180^{\prime \prime} \times 149^{\prime \prime}$. g) S $209 \mathrm{at} 613 \mathrm{MHz}$. The angular resolution is $10^{\prime \prime} \times 10^{\prime \prime}$. h) S 209 at $236 \mathrm{MHz}$. The angular resolution is $25^{\prime \prime} \times 25^{\prime \prime}$. i) $613 \mathrm{MHz}$ image of S $209 \mathrm{made}$ using $u-v$ range only up to $1 \mathrm{k} \lambda$. The angular resolution is $160^{\prime \prime} \times 136^{\prime \prime}$.

temperature of $8350 \pm 1600 \mathrm{~K}$, derived for the core of S 206 (knot-A in Fig. 1c) is in reasonable agreement with previous measurements, viz. $8400 \pm 800 \mathrm{~K}$ obtained using the H94 $\alpha$ recombination line by Carral et al. (1981), and $9118 \mathrm{~K}$ obtained from the $[\mathrm{O} \mathrm{III}] \lambda \lambda 4363,5007$ lines ratio (Deharveng et al. $2000)$. The emission measure is $3.93( \pm 0.40) \times 10^{5} \mathrm{~cm}^{-6} \mathrm{pc}$, consistent with the value obtained by Deharveng et al. (1976). For S 209, the electron temperature corresponding to the peak radio emission at $613 \mathrm{MHz}$ is estimated to be $10855 \pm 3670 \mathrm{~K}$, somewhat higher than the value of $8280 \mathrm{~K}$ obtained using the $\mathrm{H} 137 \beta$ recombination line by Churchwell et al. (1978) but in reasonable agreement with the estimate of $11000 \mathrm{~K}$ which was derived from $\mathrm{H} 91 \alpha$ \& $\mathrm{H} 114 \beta$ recombination lines (Balser et al. 1994).

Figure 3 is a plot of the electron temperature vs. Galactocentric distance for the three $\mathrm{H}_{\text {II }}$ regions studied in this paper. The solid line is the relationship obtained by Deharveng et al. (2000) from a sample of six H II regions spanning Galacto-centric distances from 6.6 to $14.8 \mathrm{kpc}$. The data for S 209 shows that this relationship appears to be valid even out to Galacto-centric distance of $\sim 18 \mathrm{kpc}$.

If there are systematic radial temperature gradients within the cores of $\mathrm{H}_{\mathrm{II}}$ regions, the $T_{\mathrm{e}}$ estimated by various methods, viz., radio continuum, RRLs, and [O III] $\lambda \lambda 4363$, 

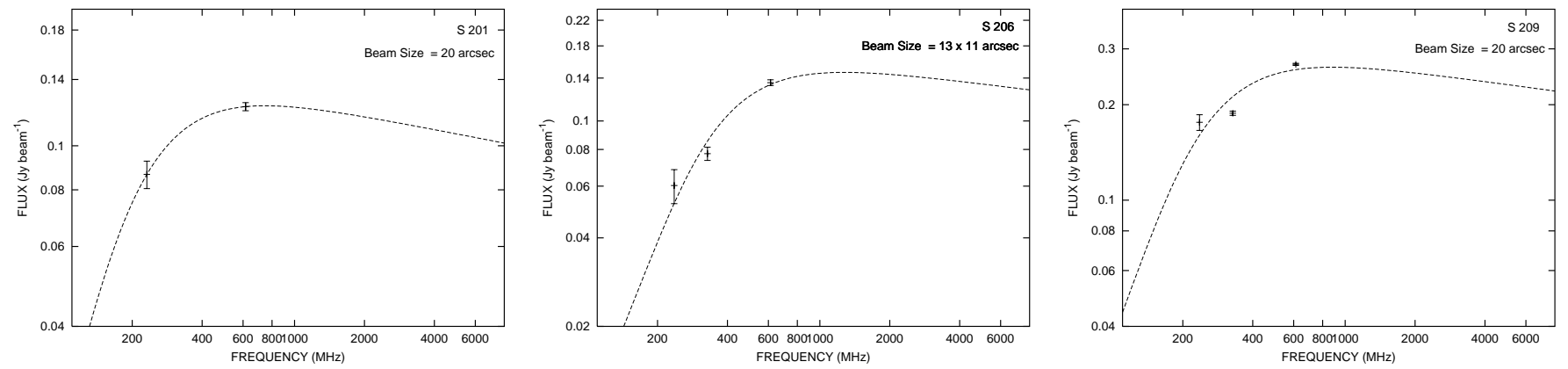

Fig. 2. Model and observed fluxes for the cores of the H II regions observed at the GMRT. The estimated emission measures and electron temperatures are listed in Table 2.

5007 lines ratio (all of which probe different physical regions) would be discordant. The radio continuum observations at low frequencies are more sensitive to outermost regions of the core of the nebula (due to the high optical depths at these frequencies). The temperature estimates from high frequency RRLs (which is where most of the observations exist) are weighted toward regions of low temperature. Finally, estimates of $T_{\mathrm{e}}$ from the $\left[\mathrm{O}_{\mathrm{III}}\right]$ lines ratio are expected to be weighted toward high temperature regions due to the high cooling rate provided by [O III] lines. Since, our estimates of $T_{\mathrm{e}}$ are in general consistent with those obtained from RRLs as well as from [O III] lines, any temperature gradients within the cores of these $\mathrm{H}$ II regions must be smaller than the combined uncertainties in these different $T_{\mathrm{e}}$ measurements. Similar concordance between $T_{\mathrm{e}}$ measured using these different methods have been obtained for W 51 (Subrahmanyan \& Goss 1995) and M 17 (Subrahmanyan \& Goss 1996; Wilson et al. 1997). Several authors have discussed the possibility of small scale temperature fluctuations in the cores of H II regions (Peimbert 1967; Rubin et al. 1998 and references therein), these cannot be ruled out based on our observations alone).

\section{Conclusions}

Three outer galaxy H II regions, S 201, S 206 and S 209 have been imaged at meter wavelengths using the GMRT. The images of these $\mathrm{H}_{\text {II }}$ regions have been obtained at a resolution of less than a pc. This is the highest resolution achieved for any $\mathrm{H}_{\text {II }}$ region at such low radio frequencies. All three $\mathrm{H}$ II regions show structures down to our resolution limit. The high resolution images near $610 \mathrm{MHz}$ of these $\mathrm{H}$ II regions show a good correspondence with the radio continuum images at $\mathrm{cm}$ wavelengths. The low resolution radio images show that these $\mathrm{H}$ II regions are surrounded by large diffuse envelopes. The high resolution radio images have allowed us to get estimates of $T_{\mathrm{e}}$ of these $\mathrm{H}_{\text {II }}$ regions. From these measurements we find that:

(1) the estimates of $T_{\mathrm{e}}$ are in general consistent with that obtained from RRLs and [O III] $\lambda \lambda 4363,5007$ line measurements, and

(2) the measured temperatures are consistent with a linear increase of $T_{\mathrm{e}}$ with Galacto-centric distance until $R_{\mathrm{G}} \sim 18 \mathrm{kpc}$.

Acknowledgements. We thank the staff of the GMRT that made these observations possible. The GMRT is run by the National Centre for

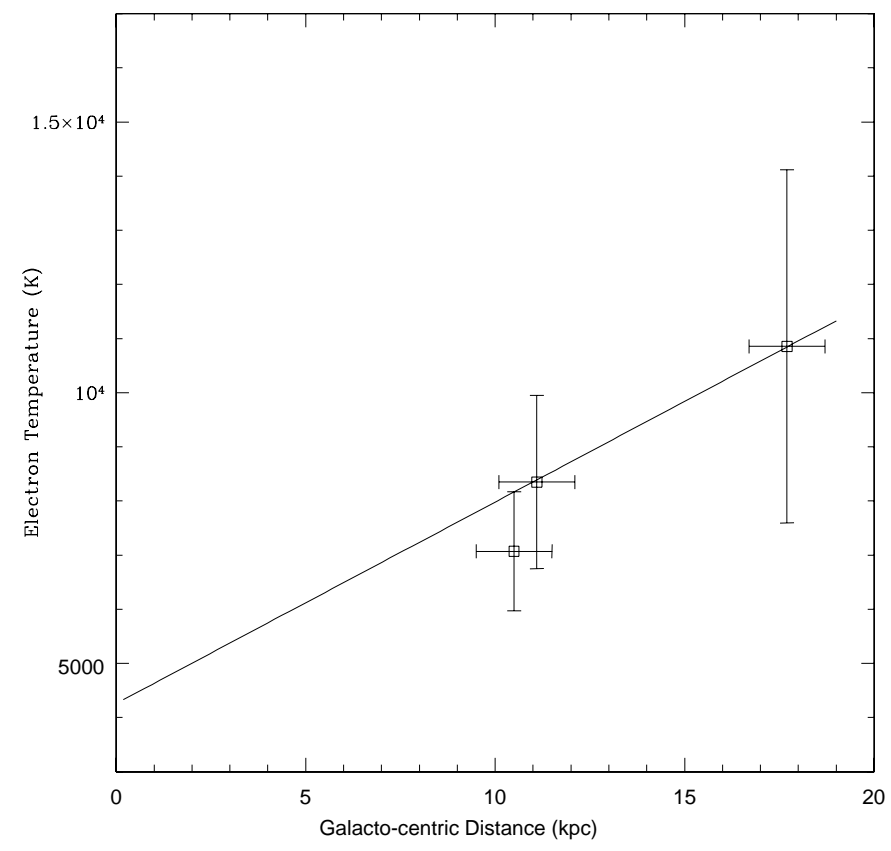

Fig. 3. The electron temperature vs. Galacto-centric distance for three $\mathrm{H}$ II regions studied in this paper. The solid line is the relationship derived by Deharveng et al. (2000) based on a sample of six $\mathrm{H}_{\text {II }}$ regions spanning a Galacto-centric distance from 6.6 to $14.8 \mathrm{kpc}$.

Radio Astrophysics of the Tata Institute of Fundamental Research. We are grateful to K. S. Dwarakanath and R. N. Mohan for useful discussions. We thank (late) K. R. Anantharamaiah for useful suggestions and comments on an earlier version of this paper. We are also grateful to one of the referees, L. Deharveng, for many comments which greatly improved the contents and readability of this paper. AO thanks NCRA-TIFR for providing hospitality during his stay at NCRA.

\section{References}

Baars, J. W. M., Genzel, R., Pauliny-Toth, I. I. K., \& Witzel, A. 1977, A\&A, 61, 99

Balser, D. S., Bania, T. M., Brockway, C. J., Rood, R. T., \& Wilson, T. L. 1994, ApJ, 430, 667

Balser, D. S., Bania, T. M., Rood, R. T., \& Wilson, T. L. 1995, ApJS, 100,371

Carral, P., Rodriguez, L. F., \& Chaisson, E. J. 1981, A\&A, 95, 388

Chini, R., \& Wink, J. E. 1984, A\&A, 139, L5 
Churchwell, E., Smith, L. F., Mathis, J., Mezger, P. G., \& Huchtmeier, W. 1978, A\&A, 70, 719

Mampaso, A., Phillips, J. P., Vilchez, J. M., Pismis, P., \& Riera, A. 1989, A\&A, 220, 235

Crampton, D., \& Fisher, W. A. 1974, Publ. Dominion Astrophys. Obs., $14(12)$

Deharveng, L., Israel, F. P., \& Maucherat, M. 1976, A\&A, 48, 63

Mezger, P. G., \& Henderson, A. P. 1967, ApJ, 147, 471

Peimbert, M. 1967, ApJ, 150, 825

Deharveng, L., Pena, M., Caplan, J., \& Costero, R. 2000, MNRAS, 311,329

Rubin, R. H., Martin, P. G., Dufour, R. J., et al. 1998, ApJ, 495, 891

Felli, M., Hjellming, R. M., \& Cesaroni, R. 1987, A\&A, 182, 313

Fich, M. 1993, ApJS, 86, 475

Fich, M., \& Blitz, L. 1984, ApJ, 279, 125

Haslam, C. G. T., Stoffel, H., Salter, C. J., \& Wilson, W. E. 1982, A\&AS, 47, 1

Icke, V., Gatley, I., \& Israel, F. P. 1980, ApJ, 236, 808

Subrahmanyan, R., \& Goss, W. M. 1995, MNRAS, 275, 75

Subrahmanyan, R., \& Goss, W. M. 1996, MNRAS, 281, 239

Swarup, G., Ananthakrishnan, S., Kapahi, V. K., et al. 1991, Curr. Sci., 60,95

Walmsley, C. M., Churchwell, E., Kazes, I., \& Le Squeren, A. M. 1975, A\&A, 41, 121

Wilson, T. L., Filges, L., Codella, C., Reich, W., \& Reich, P. 1997, A\&A, 327, 1177 